\title{
Probing the future of correlative microscopy
}

\author{
Lucy Collinson $^{1} \cdot$ Paul Verkade $^{2}$
}

Published online: 30 September 2015

(C) Springer-Verlag Berlin Heidelberg 2015

Instrumental developments in microscopy, such as confocal scanning light microscopy and super resolution light microscopy, have firmly established imaging as a key technology in modern life science research. However, this would not have been possible without the availability of the right probes - the concurrent emergence of green fluorescent protein (GFP) completely transformed the field. We can surely assume that GFP and its derivatives have now penetrated most biomedical research labs that use imaging as a tool. The importance of fluorescent probes in light microscopy can further be measured by the award of the Nobel Prize for Chemistry in 2008 to Tsien, Shimomura and Chalfie.

The role of probes is as pertinent in the emerging field of correlative microscopy, though arguably far more complex. In correlative microscopy, two or more imaging modalities are applied to a single sample, with the combined images yielding more information than when each modality is used independently. To reflect this, we often use the phrase ' $1+1=3$ '. The most established correlative microscopy technique is correlative light electron microscopy (CLEM). Usually confocal LM and transmission EM (TEM) are correlated, though recently there have been major developments in both light and electron microscopy leading to other combinations. Correlative light and scanning EM in particular is a growing area due to the availability of new 3D automated microscopes like the serial

Paul Verkade

bixpv@bristol.ac.uk

1 Lincoln's Inn Fields Laboratory, The Francis Crick Institute, 44 Lincoln's Inn Fields, London WC2A 3LY, UK

2 Wolfson Bioimaging Facility, Schools of Biochemistry and Physiology \& Pharmacology, Medical Sciences Building, University of Bristol, University Walk, Bristol BS8 1TD, UK block face SEM, the focused ion beam SEM, and array tomography [5].

However, whereas fluorescent molecules (either as an expression construct or through antibody or ligand coupling) are the most commonly used to detect a protein of interest in LM, this fluorescence is not directly visible in the electron microscope. Here, an electron-dense moiety such as a gold particle is generally required. Thus, the most direct way to produce a marker/probe (these terms are used interchangeably in the field and in this issue) is to couple both a fluorescent molecule and an electron-dense particle to the protein of interest. Unfortunately though, it is not that simple, and in most instances, the direct coupling of, e.g. a gold particle next to a fluorescent molecule decreases or completely masks the fluorescence emission (see, e.g. [2]). Hence, there have been major efforts to create the 'optimal' CLEM probe, one that is visible directly in the LM and EM and can be easily tagged to a protein of interest. Most likely there will never be a single optimal CLEM probe that can be used for all applications, and one has to carefully analyse which probe is best suited for each application.

This special issue deals with these latter two cases: the development of new probes and the application of a probe to a specific biological question. The issue starts with a paper by the group of John Robinson discussing possibly the oldest CLEM probe-fluoronanogold. Developed at the beginning of the 1990s, it combines a fluorescent moiety with a very small nanogold particle. As the quenching of fluorescence is strongly influenced by the size (and distance) of the gold particle to the fluorophore [2], this probe does not seem to suffer serious quenching. A disadvantage is that the $1.4-\mathrm{nm}$ gold is too small to be directly visible in the EM against the background contrast of cells or tissues. Hence, silver enhancement has been a necessity. Originally used on Tokuyasu sections, the application of fluoronanogold in pre-embedment 
applications has produced one of the best-established CLEM techniques (see, e.g. [3]). Indeed, we have recently made good use of the probe to elucidate the localisation and function of the ESCRTIII complex during nuclear membrane reformation following mammalian cell division [4].

Next, several related probe strategies are discussed that depend on photo- or chemical conversion of a moiety to create an electron-dense reaction product (Ellisman et al., this issue). Some of these are now well-known names in the correlative microscopy field (mini-SOG, APEX). Although they are based on a precipitation reaction and prone to diffusion of the reaction product, recent developments have resulted in a more controlled reaction and hence increased spatial resolution.

One of the markers that has the potential to be an ideal CLEM probe is the quantum dot. These small alloys, usually of cadmium and selenium, have the interesting dual properties of electron density due to the incorporation of metal, and fluorescence when excited with laser light. Notably, the emission wavelength of a quantum dot is dependent on its size and shape (the smaller ones emitting in the shorter green wavelengths, the larger towards the longer red wavelengths). However, one cannot just attach a Qdot to a protein or ligand since they have been shown to affect subcellular sorting/ trafficking [1]. Thus, getting the probe in the right location is another important issue that needs to be addressed. The groups of Bram Koster and Sander van Kasteren have recently applied 'click' chemistry to address this issue and report the latest advances here (van Elsland et al., this issue).

Whereas these papers give an overview of current probe technology, the next three papers report on the latest developments in the field, where a single moiety has the potential to be used as a dual probe. Papers by the groups of Seznec (Le Trequesser et al., this issue) and O'Toole (Morrison et al., this issue) in this issue reveal the potential of metallic particles and phosphors as correlative probes for CLEM. In a different direction, and bringing us back to the first paragraph of this introduction, a paper by the Collinson lab (Brama et al., this issue) reveals that GFP can be used as a CLEM probe in a new generation of integrated light and electron microscopes, indicating an even broader application for this transformative probe in life science research.
A CLEM experiment can generally be divided into four steps: probes, processing, imaging and analysis. Whereas the first three are covered in this issue and fall within the scope of the Journal of Chemical Biology, we have not covered the analysis part of the CLEM workflow here. This is generally the area of bioimage analysts and software engineers and involves the accurate mapping of the different images onto each other, which becomes especially important when working with single moiety markers used as dual probes for protein localisation.

It is clear from the papers in this special issue and others that the development of correlative microscopy is still in full flow and that while some technologies are well-established, others are undergoing constant improvement, and still more correlative workflows are being added as time goes by. But the availability of correlative probes will be key to the future success of such technologies, measured by their ability to answer critical life science research questions. We consider this issue a call to scientists at the biology-chemistry interface to take up the challenge of developing the next generation of correlative probes to drive life science research towards the ambitious goal of seamless patient-to-protein functional imaging.

\section{References}

1. Brown E, Verkade P (2010) The use of markers for correlative light electron microscopy. Protoplasma 244:91-97

2. Kandela IK, Albrecht RM (2007) Fluorescence quenching by colloidal heavy metals nanoparticles: implications for correlative fluorescence and electron microscopy studies. Scanning 29:152-161

3. Marra P, Maffucci T, Daniele T, Di Tullio G, Ikehara Y, Edward KL, Chan AL, Beznoussenko G, Mironov A, De Matteis MA (2001) The GM130 and GRASP65 Golgi proteins cycle through and define a subdomain of the intermediate compartment. Nat Cell Biol 3:11011113

4. Olmos Y, Hodgson L, Mantell J, Verkade P, Carlton JG (2015) ESCRT-III controls nuclear envelope reformation. Nature 522:236239

5. Peddie CJ, Collinson LM (2014) Exploring the third dimension: volume electron microscopy comes of age. Micron 61:9-19 